How often are chaotic saddles nonhyperbolic?

This article has been downloaded from IOPscience. Please scroll down to see the full text article. 1993 Nonlinearity 6779

(http://iopscience.iop.org/0951-7715/6/5/007)

View the table of contents for this issue, or go to the journal homepage for more

Download details:

IP Address: 137.132.123.69

The article was downloaded on 23/06/2010 at 07:56

Please note that terms and conditions apply. 


\title{
How often are chaotic saddles nonhyperbolic?
}

\author{
Ying-Cheng Laiłt 1 , Celso Grebogi†§, James A Yorke§ and Ittai Kan\| \\ $\dagger$ Laboratory for Plasma Research, University of Maryland, College Park, MD 20742, USA \\ $\ddagger$ Department of Physics and Astronomy, University of Maryland, College Park, MD 20742, \\ USA \\ $\S$ Department of Mathematics and Institute for Physical Science and Technology, University \\ of Maryland, College Park, MD 20742, USA \\ || Department of Mathematics, George Mason University, Fairfax, VA 22030, USA
}

Received 9 April 1992, in final form 25 January 1993

Recommended by P Grassberger

\begin{abstract}
In this paper, we numerically investigate the fraction of nonhyperbolic parameter values in chaotic dynamical systems. By a nonhyperbolic parameter value we mean a parameter value at which there are tangencies between some stable and unstable manifolds. The nonhyperbolic parameter values are important because the dynamics in such cases is especially pathological. For example, near each such parameter value, there is another parameter value at which there are infinitely many coexisting attractors. In particular, Newhouse and Robinson proved that the existence of one nonhyperbolic parameter value typically implies the existence of an interval ('a Newhouse interval') of nonhyperbolic parameter values. We numerically compute the fraction of nonhyperbolic parameter values for the Henon map in the parameter range 'where there exist only chaotic saddles (i.e., nonattracting invariant chaotic sets). We discuss a theoretical model which predicts the fraction of nonhyperbolic parameter values for small Jacobians. Two-dimensional diffeomorphisms with similar chaotic saddles may arise in the study of Poincaré return map for physical systems. Our resuits suggest that (1) nonhyperbolic chaotic saddles are common in chaotic dynamical systems; and (2) Newhouse intervals can be quite large in the parameter space.
\end{abstract}

AMS classification scheme numbers: $58 \mathrm{Fxx}$

\section{Introduction}

Chaotic saddles (in the plane) are closed, bounded, nonattracting, chaotic invariant sets with a dense orbit such that each point of the set has a stable direction and an unstable direction [1]. Chaotic saddles arising in nonlinear dynamical systems can be either nonhyperbolic or hyperbolic. For hyperbolic chaotic saddles, all the angles between the stable and unstable directions are uniformly bounded away from zero. If the angles are not bounded away from zero, we say the chaotic saddle is nonhyperbolic.

A fairly typical example of a chaotic saddle occurs in the forced damped pendulum system [2]

T Present address: Department of Biomedical Engineering, The Johns Hopkins University School of Medicine, Baltimore, MD 21205, USA. 

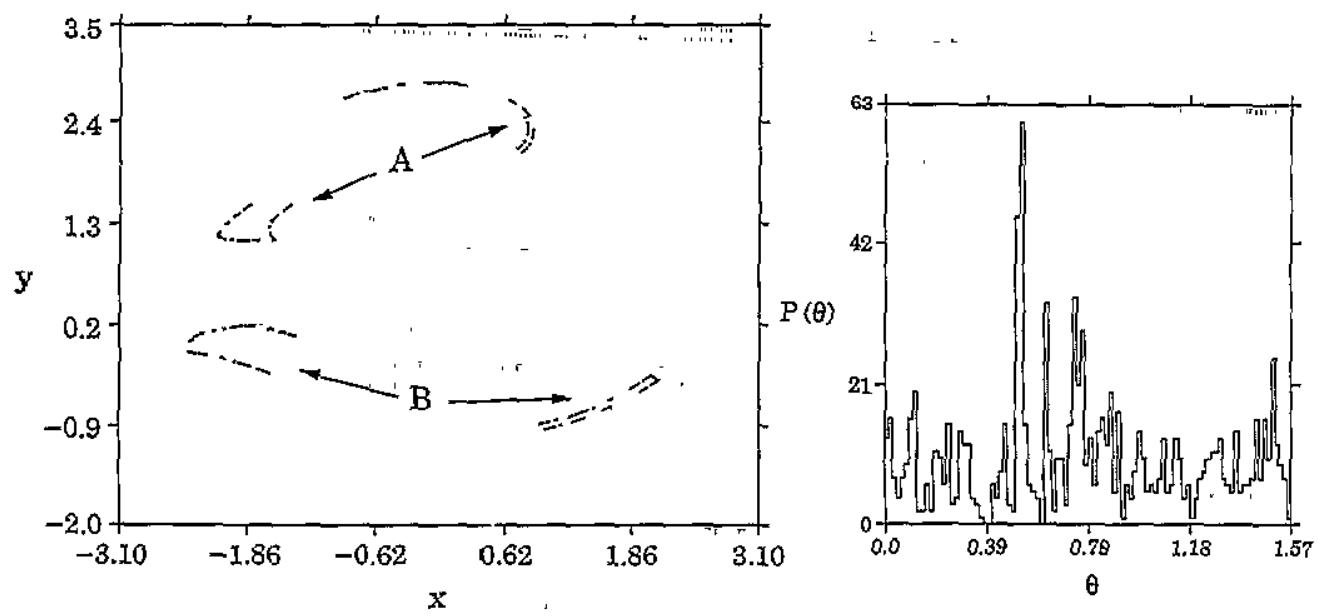

Figure 1. For the forced damped pendulum system of equation (1), (a) the nonhyperbolic chaotic saddles $A$ and $B$, and $(b)$ the histogram of 1000 angles on the chaotic saddle $A$, that is, the angle between stable and unstable manifolds from a numerical trajectory of 1000 points lying on $A$. The minimum angle observed here is about 0.01 .

$$
\begin{aligned}
& \frac{\mathrm{d} x}{\mathrm{~d} t}=y \\
& \frac{\mathrm{d} y}{\mathrm{~d} t}=-0.2 y-\sin x+2 \cos (z) \\
& \frac{\mathrm{d} z}{\mathrm{~d} t}=1 .
\end{aligned}
$$

For this system, the Poincare return map has two fixed point attractors and a number of chaotic saddles, two of which are shown in figure $1(a)$. Such sets are typically Cantor sets. We compute a trajectory on the chaotic saddle that is labelled $A$ in figure $1(a)$ and at each trajectory point we compute the angle between the stable and unstable directions. Figure $1(b)$ shows a histogram of the angle, which suggests that the chaotic saddle at this parameter value is nonhyperbolic because the minimum angle between the stable and unstable directions may be arbitrarily close to zero as one examines longer and longer segments of the trajectory. Whether it goes to zero is uncertain and further experiments are difficult because of the difficulty of making these computations for a system of differential equations. Maps such as the Hénon map are computationally much more tractable, and the errors in iterating are smaller. Figures $2(a)$ and $2(b)$ show the chaotic saddle and the histogram of angles for the Hénon map:

$$
\left(x_{n+1}, y_{n+1}\right)=\left(a-x_{n}^{2}+b y_{n}, x_{n}\right)
$$

where $a=1.6$ and $b=0.3$. Figure $2(b)$ suggests that the chaotic saddle in this case is also nonhyperbolic. For the Hénon map at $a=3.0$ and $b=0.3$, the chaotic saddle is hyperbolic, as suggested by figures $3(a)$ and $3(b)$, where the minimum angle between the stable and unstable directions is about 0.57 . Histograms like figures $1(b)$ and $2(b)$ show a complicated structure without much regularity near $\theta=0$. This irregularity persists even with much longer trajectories, that is, with much more data included in the histogram. This lack of regularity makes it impossible to extrapolate reliably to very small angles. 

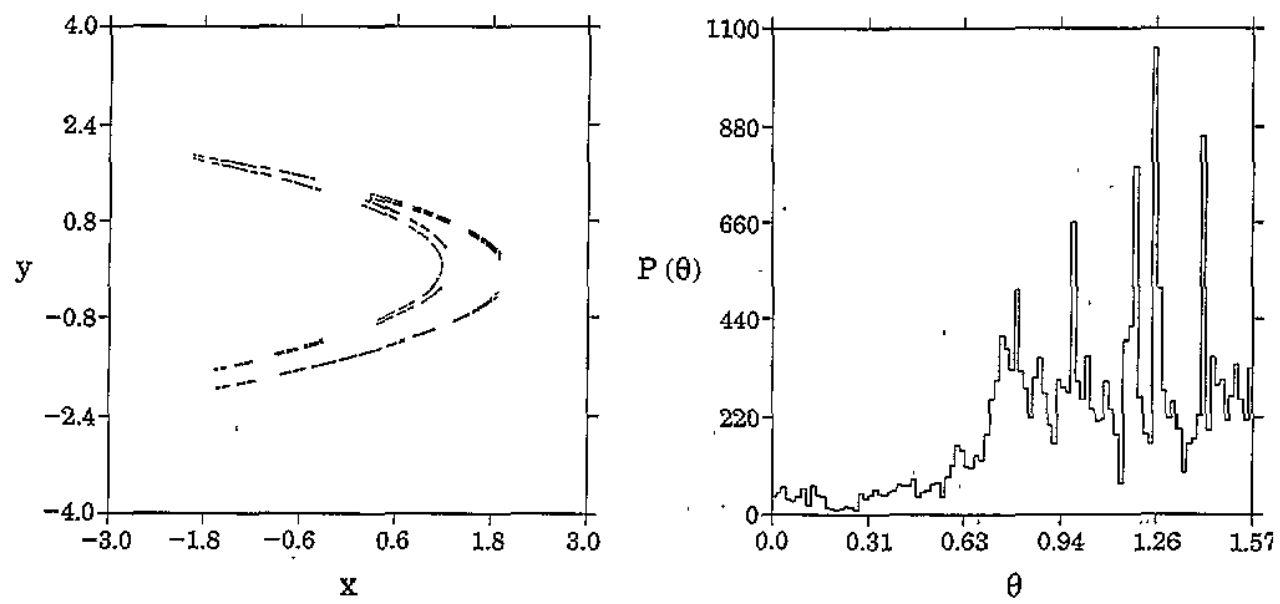

Figure 2. For the Henon map of $a=1.6$ and $b=0.3$, (a) the nonhyperbolic chaotic saddle, and $(b)$ a histogram of 20000 angles on the chaotic saddle.
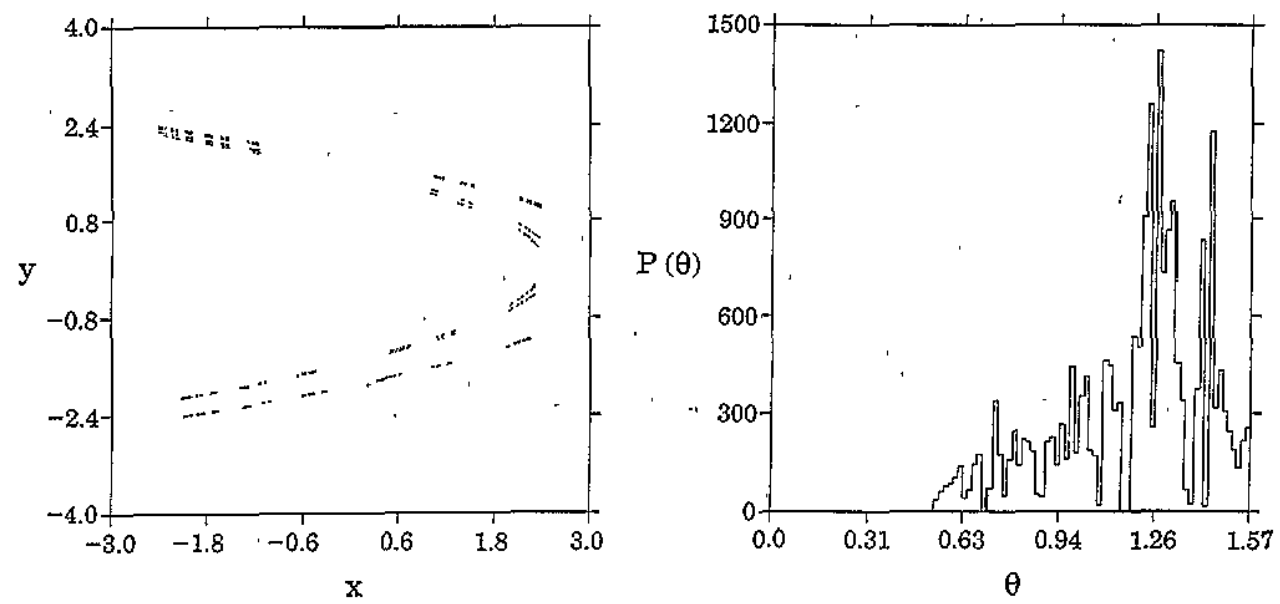

Figure 3. For the Henon map of $a=3.0$ and $b=0.3$, (a) the hyperbolic chaotic saddle, and (b) a histogram of 20000 angles on the chaotic saddle. Note that the angles are bounded awray from 0 .

The objective of this paper is to examine how often nonhyperbolic chaotic saddles occur in nonlinear dynamical systems. Newhouse [3] and Robinson [4] proved that for a large class of two-dimensional dissipative dynamical systems, the existence of one nonhyperbolic parameter value implies the existence of an interval of such nonhyperbolic parameter values (we call such an interval a Newhouse interval). Hence, if we vary the parameters over an interesting finite range, the fraction of the set of nonhyperbolic parameter values is nonzero. We have developed an efficient numerical procedure to estimate this fraction of nonhyperbloic parameter values. Our computation suggests that this fraction is quite significant and, therefore, nonhyperbolic chaotic saddles are apparently common in chaotic dynamical systems. Furthermore, our computation also suggests that the Newhouse intervals can be rather large in parameter space. We primarily investigate the Hénon map, in the 
parameter range where there is no attractor. Although our computation is for the Henon map, we believe that nonhyperbolic chaotic saddles occur even more often in physical systems such as the forced damped pendulum system.

There are many reasons why it is necessary to know how often dynamical systems are nonhyperbolic. (i) Since most of the results in the theory of dynamical systems have been proved for hyperbolic systems, it is desirable to know whether a particular system we are dealing with is hyperbolic or not. For example, the shadowing lemma (which tells us whether there is a true trajectory in the neighbourhood of a computer-generated trajectory) only holds for hyperbolic systems $[5,6]$. Indeed, shadowing is usually impossible for nonhyperbolic invariant sets. Another example is the unstable periodic orbit theory for multifractal characterization of chaotic systems [7] which only applies for hyperbolic systems. (ii) For the application of particular numerical techniques to chaotic trajectories, it is often important to determine the extent to which the chaotic trajectories avoid tangencies between stable and unstable manifolds. For example, the computer-aided proof of the finite time shadowing property [8-10] for nonhyperbolic systems relies on the fact that the particular trajectory investigated avoids tangencies between stable and unstable manifolds. Another example arises in the context of noise reduction technique for chaotic systems, which is important in dealing with experimental data. An efficient noise reduction scheme discussed by Hammel [11] for chaotic dynamical system depends on the trajectory being hyperbolic. For trajectory points near tangencies of stable and unstable manifolds, this scheme may yield results with dramatic errors.

We now preview our method for determining the fraction and distribution of the nonhyperbolic parameter values for the Hénon map. For each parameter $(a, b)$ of interest, we find a single trajectory on the chaotic saddle by using the 'PIM-triple' method [12] mentioned in the appendix. The chaotic saddles we examine are unstable. Trajectories near them tend to diverge rapidly. The PIM-triple method produces numerical trajectories of a map $\boldsymbol{F}$, that is, $\boldsymbol{x}_{n+1}=\boldsymbol{F}\left(\boldsymbol{x}_{n}\right)+\varepsilon_{n}$, where the errors $\varepsilon_{n}$ move the trajectories back to the chaotic saddle (or more precisely, back to the stable manifold of the chaotic saddle). The resulting trajectory seems to be typical within the class of trajectories that remains near the saddle. For the Henon map, almost all trajectories diverge to $\infty$ for most of the parameter values we examine. The chaotic saddle we see are 'relative' attracting, that is, they are attractors for the class of trajectories that remain bounded as $n \rightarrow \infty$. Chaotic saddles which are not relative attractors may be common, but they cannot be seen by the PIMtriple method. In our experience with the Hénon map, initializing the PIM-triple method differently produce different numerical trajectories that sketch out the same relative attractor. We note that the PIM-triple method has a rigorous justification only for hyperbolic chaotic saddles [12].

We test the minimum angle between the stable and unstable directions along the trajectory on the chaotic saddle. Our numerical studies with multiple initial conditions indicate that the results do not depend on the initial point. We find that if one chaotic trajectory on the chaotic saddle is nonhyperbolic, then typically they all are. In general, the angle between the stable and unstable directions is defined as follows. Each point on the chaotic saddle has a stable direction and an unstable direction, which are the local tangent vectors of the stable and unstable manifolds of that point, respectively. We say $s$ is the stable direction for a map $T$ at $x$ if there is a constant $K<1$ such that $\left|D T^{n}(x)(s)\right| \leqslant K^{n}|s|$ as $n \rightarrow \infty$. We say $u$ is the unstable direction at $x$ if $\left|D T^{-n}(x)(u)\right| \leqslant K^{n}|u|$ as $n \rightarrow \infty$. We let $\theta(x, a, b) \in[0, \pi / 2]$ be the angle between the stable the unstable directions at $x$, where $\boldsymbol{x}$ is a point of the invariant set. Note that for rare points $\boldsymbol{x}$, it is possible for $\boldsymbol{s}$ and $u$ to be identical so $\theta(\boldsymbol{x}, a, b)$ would be zero. For a trajectory $x_{n+1}=T\left(x_{n}, a, b\right)$, 
We define the lower bound of the angle to be $\theta_{\text {inf }}\left(x_{0}, a, b\right) \equiv \inf _{i=0,1,2, \ldots, \infty} \theta\left[T^{i}\left(x_{0}\right), a, b\right]$, where $x_{0}$ is the initial condition. Since we investigate only a finite number of iterates, we study $\theta_{m}\left(x_{0}, a, b, N\right)=\min _{i=0,1,2, \ldots, N} \theta\left[T^{i}\left(x_{0}\right), a, b\right]$. While $\theta\left[T^{i}\left(x_{0}\right), a, b\right]$ is the angle at a point, $\theta_{m}\left(x_{0}, a, b, N\right)$ is the minimum of the angle for $(N+1)$ points of the trajectory. Since our investigation indicates negligible dependence on initial data, we ignore the dependence of $\theta_{\text {inf }}\left(x_{0}, a, b\right)$ or $\theta_{m}\left(x_{0}, a, b\right)$ on the initial point $x_{0}$ and write $\theta_{\mathrm{inf}}(a, b)$ or $\theta_{m}(a, b, N)$, where $\theta_{\mathrm{inf}}(a, b)=\operatorname{Iim}_{N \rightarrow \infty} \theta_{m}(a, b, N)$. We believe that the procedure we have developed allows us to test with reasonable reliability whether $\theta_{\text {inf }}(a, b)$ is 0 , despite the fact that we examine only upto $10^{7}$ iterates of a trajectory. Though uncertainties remain in individual cases, we feel we can reliably report the fraction of the parameter values studied that are nonhyperbolic. See section 4 for a discussion of our criterion.

Our main results concern a study of the Hénon map for an interesting set of parameter values $(a, b)$. Specifically, we restrict $b \in(0,1)$. Given $b$, let $a_{\mathfrak{c}}(b)$ be the crisis value, the value at which the main attractor is destroyed (the attractor collides with the basin boundary [1]), and let $a_{\mathrm{h}}(b)$ be the 'last tangency value' so that for $a>a_{\mathrm{h}}(b)$, the chaotic saddle is hyperbolic. Figure 4(a) shows, schematically, the nature of various chaotic invariant sets in different ranges of parameter $a$ for a given $b$ value. Figure $4(b)$ shows the boundary of the parameter regime of interest [namely, the $a_{\mathrm{c}}(b)$ and $a_{\mathrm{h}}(b)$ curves]. We investigate $a$ between $a_{\mathrm{c}}(b)$ and $a_{\mathrm{h}}(b)$. In this interval, the fraction of nonhyperbolic parameter values for most values of $b$ is larger than 0.2 and the maximum length of the Newhouse interval is the order of $10^{-1}$. We furthermore find that the nonhyperbolic fraction of $\left[a_{c}, a_{\mathrm{h}}\right]$ tends to 1 as $b \rightarrow 0$. A theoretical model explaining the small $b$ results is given in section 5 . Our major result is shown in figure 5 , where we plot the numerically calculated nonhyperbolic parameter values for $b \in(0,1)$ and $a \in\left[a_{\mathrm{c}}(b), a_{\mathrm{b}}(b)\right]$. Altogether, 70000 parameter pairs $(a, b)$ have been tested in the region shown in figure $4(b)$ between the curves $a_{\mathrm{c}}$ and $a_{\mathrm{h}}$.

Of them about $0.9 \%$ result in attractors.
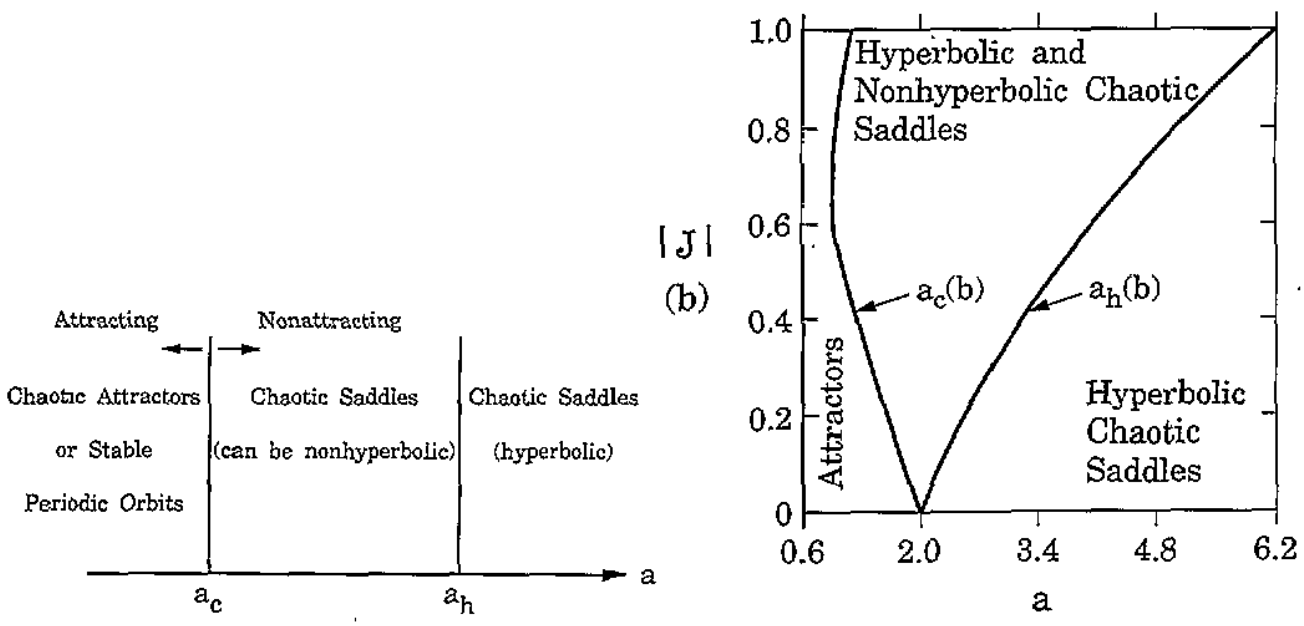

Figure 4. Parameter regime that we investigate. $(a)$ For a given $b$ value, the nature of various chaotic invariant sets in different ranges of $a$. (b) The parameter boundaries of interest the $a_{\mathrm{c}}(b)$ and $a_{\mathrm{h}}(b)$ curves]. For $a<a_{\mathrm{c}}(b)$, there are chaotic attractors, while for $a>a_{\mathrm{h}}(b)$, the chaotic saddles are hyperbolic.

The organization of this paper is as follows. In section 2 , we briefly review the Newhouse lemma. In section 3, we describe our method to calculate angles between the 


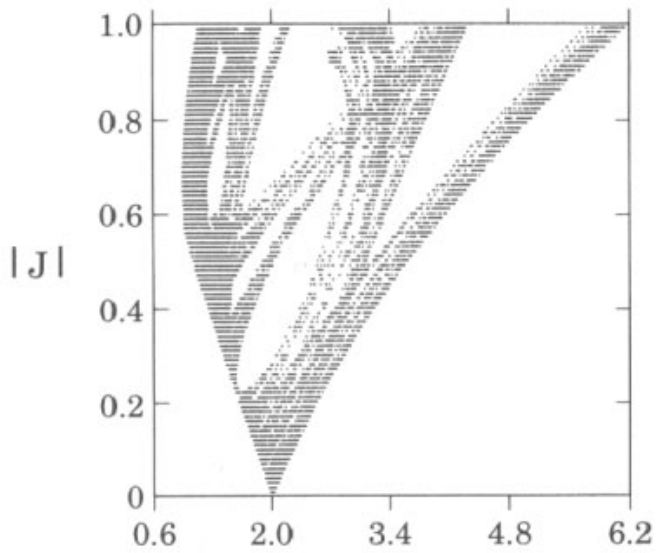

Figure 5. Hénon map, set of parameter values with nonhyperbolic chaotic saddles. An average of 700 values of $a$ were tested for each of 100 values of b. As discussed in section 4 , about $33 \%$ of the nonhyperbolic parameter pairs are missed and about $11 \%$ are incorrectly plotted (see section 4 for details). These errors should have virtually no effect on the overall configuration shown above. The left and right edges of this set coincide with the curves shown in figure $4(b)$. (a)

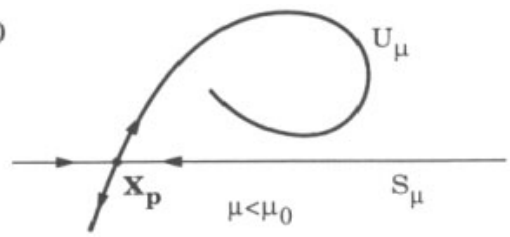

(b)

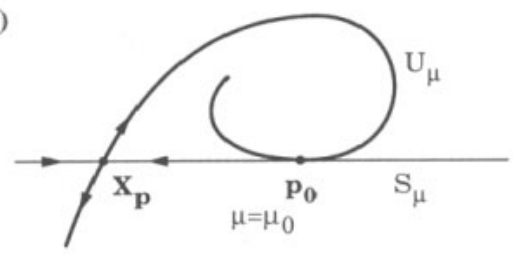

(c)

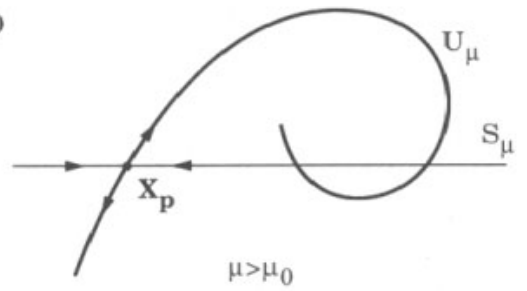

Figure 6. The stable and unstable manifolds of a saddle point $x_{p}$ for $(a) \mu<\mu_{0},(b) \mu=\mu_{0}$. and $(c) \mu>\mu_{0}$.

stable and unstable directions for points on the chaotic saddle. In section 4 , we discuss our criterion to detect the nonhyperbolic parameter values. In section 5 we present a theoretical model which explains why the nonhyperbolic fraction of $\left[a_{\mathrm{c}}, a_{\mathrm{h}}\right]$ tends to 1 as $b$ goes to 0 . In section 6, we present our results for the Hénon map.

\section{The Newhouse Iemma}

We consider the following one-parameter family of two-dimensional maps:

$$
x_{n+1}=T\left(x_{n} ; \mu\right)
$$

where $\boldsymbol{x}$ is in the plane, $\mu$ denotes the parameter to be varied and the function $T$ is threetimes continuously differentiable. We assume that $T$ has a periodic point $x_{p}$ of period $n$, i.e., $x_{p}$ is invariant under the map $T: T^{n}\left(x_{p}, \mu\right) \equiv x_{p}$. We assume that the periodic point $x_{p}$ is a saddle point, i.e., the eigenvalues of the planar linear map $D T\left(x_{p}\right)$ satisfy $\left|\lambda_{2}\right|<1<\left|\lambda_{1}\right|$. Newhouse assumes $\left|\lambda_{1} \lambda_{2}\right|<1$. The one-dimensional stable manifold of $x_{p}, S_{\mu}$, is the set of points $y$ such that $\left\|T^{n}(y, \mu)-x_{p}\right\| \rightarrow 0$ as $n \rightarrow \infty$. The one-dimensional unstable 
manifold of $x_{p}, U_{\mu}$, is the set of points $z$ such that $\left\|T^{-n}(z, \mu)-x_{p}\right\| \rightarrow 0$ as $n \rightarrow \infty$. As the parameter $\mu$ varies, $S_{\mu}$ can be tangent to $U_{\mu}$ at some $p_{0}$. A typical situation is shown in figure 6. For $\mu<\mu_{0}, U_{\mu}$ is away from $S_{\mu}$; for $\mu=\mu_{0}, U_{\mu}$ is tangent to $S_{\mu}$ at $p_{0}$; and for $\mu>\mu_{0}, U_{\mu}$ intersects $S_{\mu}$ transversally. Furthermore, if the following two conditions are satisfied: (i) the tangency is quadratic and (ii) the tangency is reached smoothly as $\mu$ varies through $\mu_{0}$, i.e., the rate of approach of the two manifolds is bounded away from zero, then we say that the tangency of $S_{\mu}$ and $U_{\mu}$ for $\mu=\mu_{0}$ is nondegenerate. We call $\mu_{0}$ a nondegenerate tangency value. For such nondegenerate tangencies, Newhouse [3] and Robinson [4] proved the following lemma:

Let $\mu_{0}$ be a nondegenerate tangency value for $T$. Then for every $\varepsilon>0$ there is $a$ nontrivial interval (positive length) $I$ in $\left(\mu_{0}-\varepsilon, \mu_{0}+\varepsilon\right)$ such that I contains a dense set of nondegenerate tangency values. The interval $I$ does not necessarily contain $\mu_{0}$.

We call interval $I$ the Newhouse interval. This lemma tells us that one nonhyperbolic parameter value implies the existence of an interval $I$ of nonhyperbolic parameter values.

\section{Angle calculation}

In this section, we present our numerical method for calculating angles between stable and unstable directions for points on the chaotic set (which can be either a chaotic attractor or a chaotic saddle). The method is demonstrated with numerical examples for the Hénon map and for the forced damped pendulum system. We also discuss the convergence of our method.

\subsection{Numerical procedure for calculating the angle}

In order to detect whether a chaotic invariant set is nonhyperbolic or hyperbolic, the first step is to calculate the angles between the stable and unstable directions for points $\boldsymbol{x}$ along a trajectory on the chaotic set. Since in this section, we calculate angles for a chaotic trajectory at fixed parameter values, we denote $\theta(x) \equiv \theta(x, a, b)$. Our numerical procedure for calculating the angles is as follows. First, we calculate a single orbit on the chaotic set either by directly iterating the map $T$ (for a chaotic attractor) or by using the PMM-triple method $[12,13]$ for a chaotic saddle (cf., appendix). Second, we calculate the stable and unstable directions for each point on the chaotic set. After we find the stable and unstable directions at the point $x$, we then choose $\theta(x)$ to be the smaller of the two angles defined by the two straight lines along the stable and unstable directions at $x$. Hence, we have $0 \leqslant \theta(x) \leqslant \pi / 2$.

In order to find the stable direction at the point $x$, we first iterate this point forward under the map $N$ times and get a trajectory $T^{1}(x), T^{2}(x), \ldots, T^{N}(x)$, as schematically shown in figure $7(a)$. Now imagine we put a circle of radius $\epsilon$ (with $\epsilon$ arbitrarily small) on the point $T^{N}(x)$, if we iterate this circle backward once, the circle will become an ellipse at the point $T^{N-1}(x)$ with the major axis along the stable direction of the point $T^{N-1}(x)$. We continue iterating this ellipse backwards, while at the same time keeping the ellipse's major axis of order $\epsilon$ via some necessary normalizations. When we iterate the ellipse all the way back to the point $x$, the ellipse becomes very thin with its major axis along the 

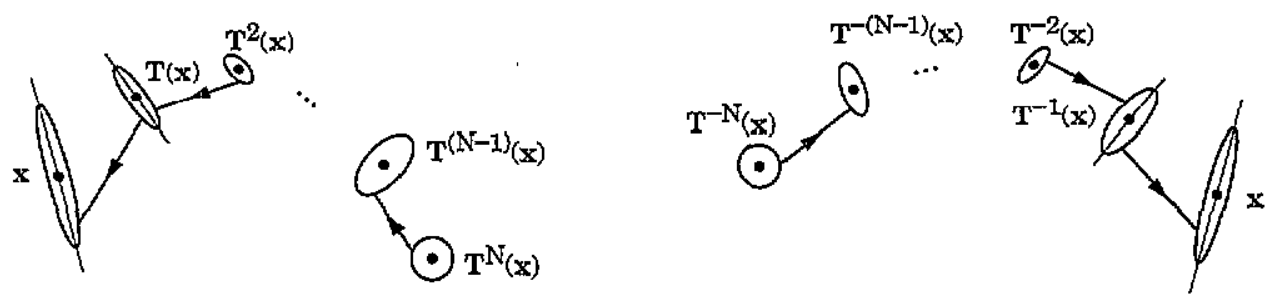

Figure 7. Schematic illustration of our numerical method to calculate (a) the stable direction, and $(b)$ the unstable direction for a point $x$ on the chaotic set.

stable direction at point $x$. This is the basic idea to calculate the stable direction at point $\boldsymbol{x}$.

In practice, instead of using a small circle, we take a unit vector at the point $T^{N}(x)$ since the Jacobian matrix of the inverse map $T^{-1}$ rotates a vector in the tangent space of $T$ towards the stable direction. Thus, we iterate a unit vector backward to point $x$ by multiplying by the Jacobian matrix of the inverse map at each point on the already existing orbit. We normalize the vector after each multiplication to the unit length. For sufficiently large $N$, the unit vector we get at point $\boldsymbol{x}$ is a good approximation of the stable direction at $x$. A key point in the calculation is that we do not actually calculate the inverse Jacobian matrix along the trajectory by iterating the point $T^{N}(x)$ backwards using the inverse map $T^{-1}$. The reason is that if we do so, the trajectory will usually diverge from the original trajectory $T^{N}(x), T^{N-1}(x), \ldots, T^{1}(x)$ after only a few backward interations. What we do is to store the inverse Jacobian matrix at every point of the orbit $T^{i}(x)(i=1, \ldots, N)$ when we iterate forward the point $\boldsymbol{x}$ beforehand.

Similarly, as shown schematically in figure $7(b)$, to find the unstable direction for point $x$ on the chaotic set, we first iterate $x$ backward under the inverse map $N$ times to get a backward orbit $T^{-j}(x)$ with $j=N, \ldots, 1$. We then choose a unit vector at point $T^{-N}(x)$ and iterate this unit vector forward to point $x$ by multiplying by the Jacobian matrix of the map $N$ times since the Jacobian matrix of the forward map rotates a vector towards the unstable direction. We normalize the vector to the unit length at each step. The final vector at point $x$ is a good approximation of the unstable direction at that point if $N$ is large enough. Again, to avoid divergence from the original trajectory, we do not actually iterate the inverse map. What we do in this case is to choose $x$ to be the end point of a forward orbit, all the points before $x$ are the inverse images of $x$ and we store the Jacobian matrix of forward map at those points.

\subsection{Numerical examples}

3.2.1. The Hénon map. For the Hénon map of equation (2), we fix $b=0.3$ and vary $a$. First, we choose $a=1.4$. For this parameter value, we have numerically verified that the map has a chaotic attractor. Figures $8(a)$ and $8(b)$ show the stable and unstable directions of 2000 points on the chaotic attractor, respectively. Both stable and unstable directions agree very well with the rigorous calculation of the stable and unstable manifolds at the corresponding trajectory points [14]. We then choose a large number of points on the chaotic attractor and calculate the angle between the stable and unstable directions at each point. Figure $8(c)$ shows the histogram of angles for 20000 points on the attractor. Figure $8(c)$ suggests that the chaotic attractor is nonhyperbolic because there exist points on the attractor at which the angles between stable and unstable directions are arbitrarily close to zero. 

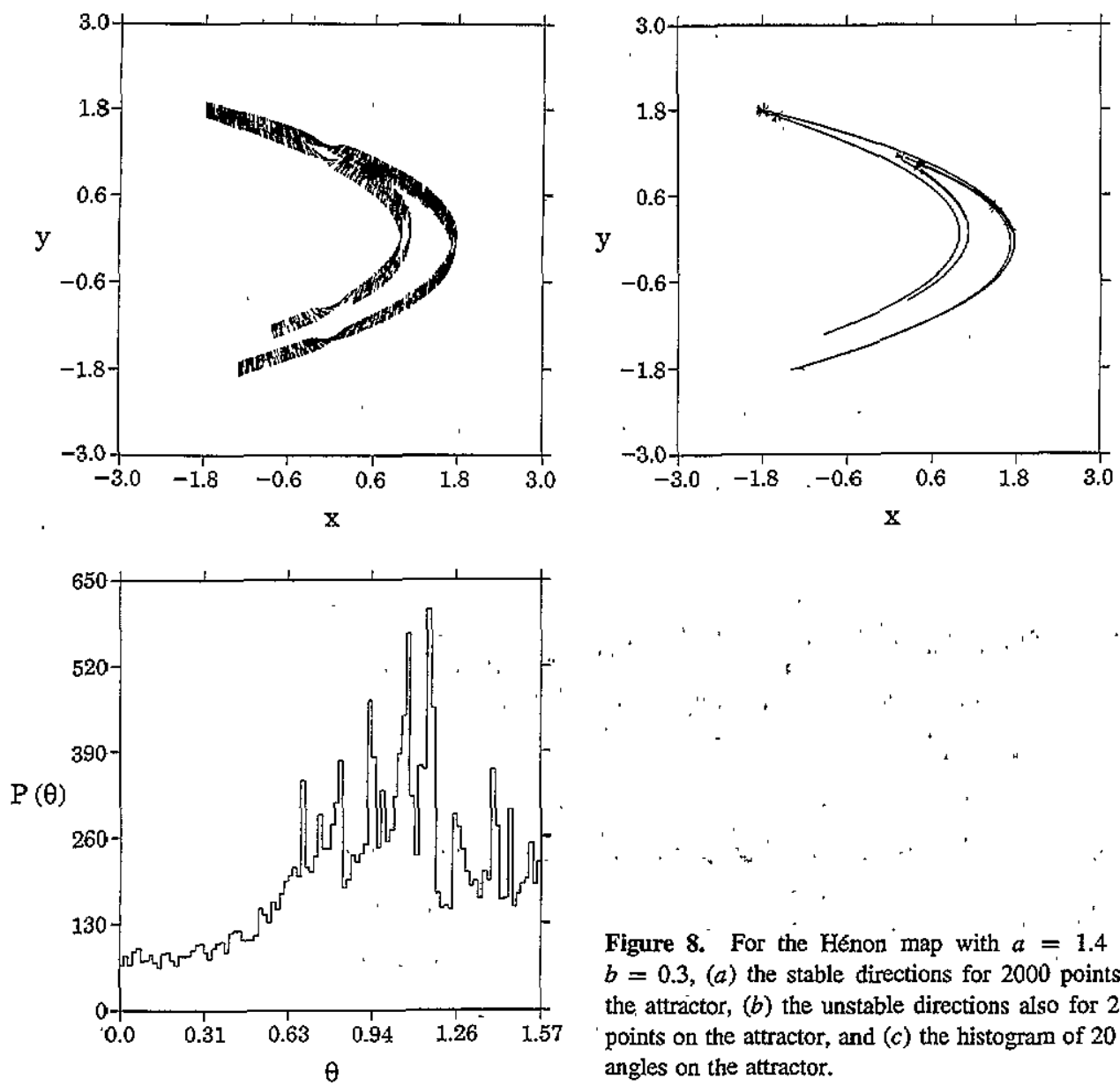

Figure 8. For the Henon map with $a=1.4$ and $b=0.3,(a)$ the stable directions for 2000 points on the attractor, $(b)$ the unstable directions also for 2000 points on the attractor, and (c) the histogram of 20000 angles on the attractor.

As we increase $a$ from $a=1.4$, a boundary crisis occurs at $a_{c} \approx 1.426$ in which the chaotic attractor is destroyed [1] and transformed into an invariant chaotic saddle for $a>a_{\mathrm{c}}$. Figure 2(a) shows the picture of the chaotic saddle at $a=1.6$, which we calculated using the PMM-triple algorithm. Figure $2(b)$ shows the histogram of 20000 points on the chaotic saddle for $a=1.6$. Figure $2(b)$ is suggestive that there exist tangency points of stable and unstable manifolds. Hence, the chaotic saddle at $a=1.6$ is nonhyperbolic.

A hyperbolic chaotic saddle can be obtained if we choose $a=3.0$. Figure $3(a)$ shows the chaotic saddle at $a=3.0$. Figures $3(b)$ shows the angle distribution for 20000 points on the chaotic saddle. It is clear from figure $3(b)$ that the angles of all the points considered are bounded away from zero and the minimum angle of these 20000 angles is approximately 0.57 .

3.2.2. The forced damped pendulum system. The forced damped pendulum system is given by equation (1). It is known that [2] for this set of parameter values, the time- $2 \pi$ map has two stable fixed points and several invariant chaotic saddles, two of which are in the basins of these two fixed points and are shown in figure $1(a)$ (denoted by $A$ and $B$ ). We take the Poincare surface of section at $t=2 n \pi, n=0,1, \ldots$, and use the PIM-triple method to calculate those chaotic saddles. 
The evolution of an infinitesimal tangent vector of a set of $N$ first order differential equations:

$$
\frac{\mathrm{d} x_{i}}{\mathrm{~d} t}=F_{i}\left(x_{1}, x_{2}, \ldots, x_{N}\right) \quad i=1,2, \ldots, N
$$

is given by:

$$
\frac{\mathrm{d} \delta \boldsymbol{x}}{\mathrm{d} t}=D \boldsymbol{F}(\boldsymbol{x}) \cdot \delta \boldsymbol{x}
$$

where $D F(x)$ is the $N \times N$ Jacobian matrix with the following matrix elements:

$$
D F_{i j}(x)=\frac{\partial F_{i}}{\partial \dot{x}_{j}} \text {. }
$$

We solve equations (4) and (5) by using the fourth order Runge-Kutta algorithm. We then calculate the local stable and unstable directions for 1000 points on each chaotic saddle $A$ and $B$ in figure $1(a)$. Figure $1(b)$ shows a histogram of 1000 angles on the chaotic saddle $A$. From figure $1(b)$, apparently there exist angles approaching zero. The histogram of angles on the chaotic saddle $B$ has similar behaviour. Therefore, both chaotic saddles for this set of parameter values for the forced damped pendulum system are nonhyperbolic. In fact, if one chaotic saddle is nonhyperbolic, the other must also be nonhyperbolic due to the symmetry $[t \rightarrow t+\pi, z \rightarrow z+\pi, x \rightarrow-x]$ in the equations.

\subsection{Convergence rate}

In this subsection, we briefly discuss the convergence rate of our method for calculating the angle. To calculate the local stable (unstable) direction for a point $x$ on the chaotic set, we need to iterate forward (backward) this point $N$ times to the point $\left.T^{N}(x)\left[T^{-N}(x)\right)\right]$ and then iterate a unit vector backward (forward) to point $x$ by evolving the Jacobian matrix. In our subsequent numerical experiments, we use $N=100$. As we will explain below, $N=20$ is quite adequate. Let $\Delta \theta_{N}^{\text {s,u }} \equiv\left\langle\Delta \theta_{N}^{\text {s,u }}(x)\right\rangle$ be the error between the calculated unit vectors at point $x$ and the local stable or unstable direction at $\boldsymbol{x}$. By convergence rate we mean the average rate at which the error decreases as $N$ increases. The average is over an ensemble of unit vectors, where one vector is calculated at each $x$, for a large number of $x$ values chosen with respect to the natural measure on the chaotic set. More specifically, we have

$$
\Delta \theta_{N}^{\mathrm{s}, \mathrm{u}}=\Delta \theta_{0} \exp \left(-\delta_{\mathrm{s}, \mathrm{u}} N\right)
$$

where $\delta_{\mathrm{s}}$ and $\delta_{\mathrm{u}}$ are the convergence rates for stable and unstable directions, respectively, and $\Delta \theta_{0}$ is the error in the initial angle.

Figures $9(a)$ and $9(b)$ show the dependence of $\Delta \theta_{N}^{s}$ and $\Delta \theta_{N}^{u}$ on $N$ on a semi-logarithmic scale, where the average is over 1000 points on the chaotic saddle for the Henon map at $a=1.6$ and $b=0.3$. With $N=10$, the error in the stable direction is about $\mathrm{e}^{-10} \sim 10^{-5}$. While with $N=20$, the error is about $10^{-8}$. The convergence rates for the stable direction $\delta_{\mathrm{s}}$ and for the unstable direction $\delta_{\mathrm{u}}$ are approximately $2.13 \pm 0.02$ and $2.07 \pm 0.02$ (least square fit), respectively. We have also calculated the convergence rate for particular points $\boldsymbol{x}$ near tangencies of the stable and unstable manifolds to verify the applicability of our numerical method in this case. For instance, for a point $x$ near such a tangency in which the angle $\theta$ between the stable and unstable manifolds is less than $1.91 \times 10^{-5}$, we found that $\delta_{s}=2.54 \pm 0.01$ and $\delta_{u}=2.15 \pm 0.02$. Therefore, we conclude that our method for calculating the angle converges quickly both for typical points on the chaotic set and for points near tangencies of stable and unstable manifolds. 

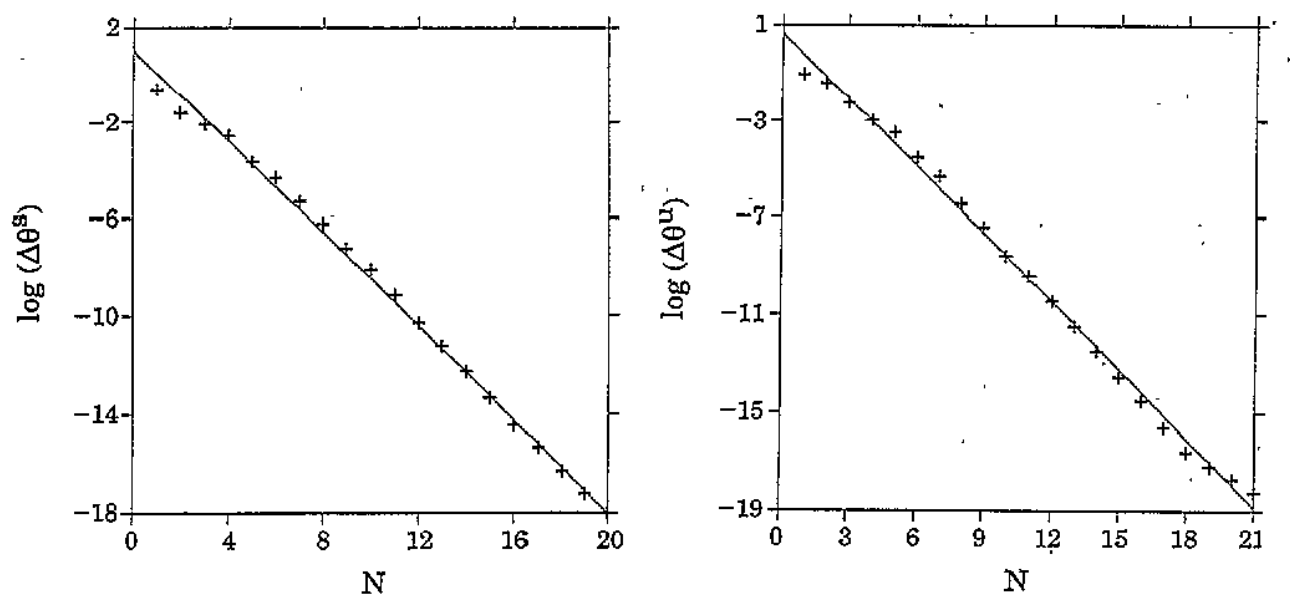

Figure 9. For the Henon map of $a=1.6$ and $b=0.3$, the average error over 1000 points on the chaotic saddle versus the number of iterates in calculating $(a)$ the stable direction, and $(b)$ the unstable direction.

\section{Criterion for detecting whether the minimum angle is zero}

In this section, we establish a criterion for detecting whether the lower bound angle $\theta_{\text {inf }}(a, b)$ for a particular parameter value $(a, b)$ is zero for the Hénon family. The parameter regime of $(a, b)$ is shown in figures $4(a)$ and $4(b)$. For the 70000 pairs $(a, b)$ tested (cf. figure 5), only about $0.9 \%$ are attractors. For the rest, the PIM-triple method is used to generate a numerical trajectory. Figure 10 shows the minimum angle $\theta_{m}(a, b, N)$ versus $a$ for $b=0.3$, where we use $N=10^{4}$ iterates for each of the 10000 tested values of $a$. In figure 10 , the diamonds plotted at height 0.5 denote parameter values at which there exist attractors. Typically, for $a>a_{\mathrm{c}}$, the basins of these attractors are small and these attractors only occur in small parameter intervals [15].

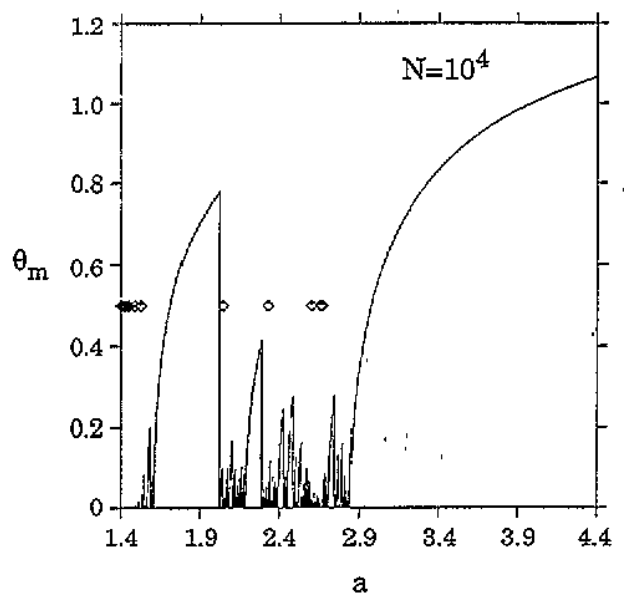

Figure 10. Henon map with $b=0.3$, a plot of the minimum angle $\theta_{m}(a, b, N)$ versus $a$, where we use $N=10^{4}$ iterates for each of the 10000 tested values of a. The diamonds plotted at height 0.5 denote parameter values at which there exist attractors. Of these 10000 tested $a$ values, about $1 \%$ result in attractors.

Our goal is to detect whether the angles $\theta_{m}(a, b, N)$ tends to zero as $N \rightarrow \infty$. We of course exclude the attractors from consideration. We calculate $\theta_{m}(a, b, N)$ for $N=10^{4}$. If 
this minimum angle is small enough, it is likely that the chaotic saddle is nonhyperbolic and, hence, $\theta_{m}(a, b, N)$ will likely go to zero as $N \rightarrow \infty$. The result of increasing the number of iterates is shown in figures $11(a)$ and $11(b)$, where $b=0.3, a \in$ [1.46.1.51], $N=10^{4}$ and $10^{5}$ for figure $11(a)$ and figure $11(b)$, respectively. On the other hand, if $\theta_{m}(a, b, N)$ is not small, then the chaotic saddle is more likely to be hyperbolic. In the hyperbolic case, it is likely that $\theta_{m}(a, b, N)$ will not decrease even if we increase $N$.
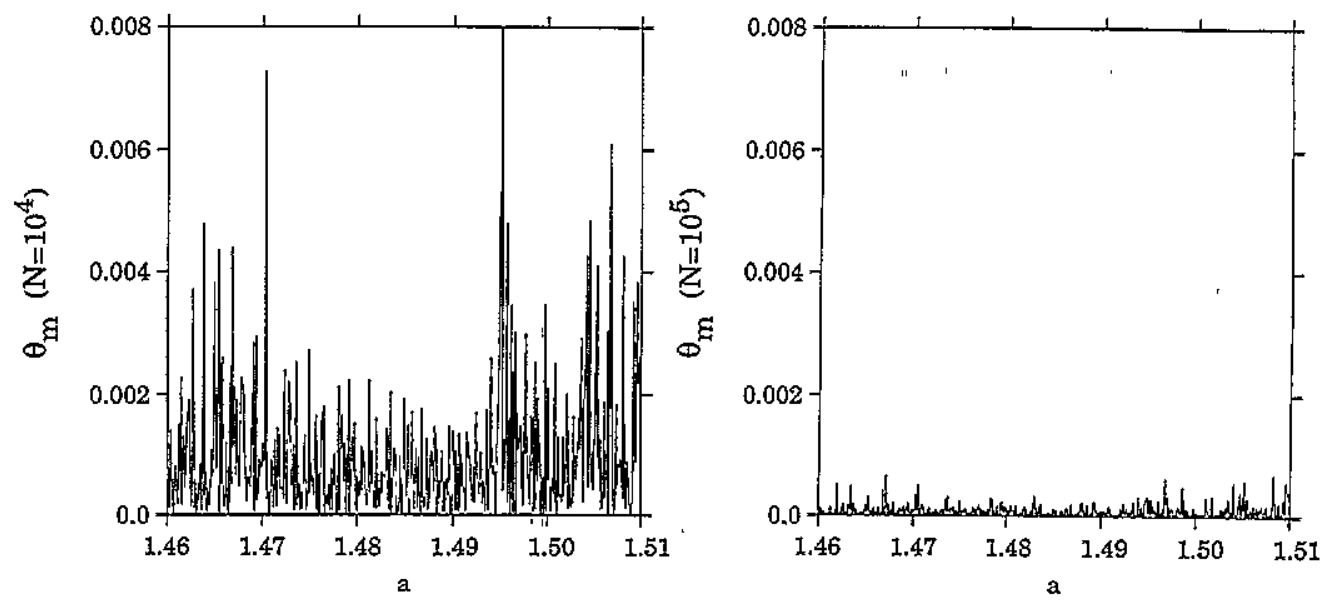

Figure 11. The effect of increasing the number of iterates on the minimum angle. For $b=0.3$ and $a \in[1.46,1.51], \theta_{m}(a, b, N)$ versus $a$ for $(a) N=10^{4}$, and $(b) N=10^{5}$.

We need to establish a criterion for deciding that a pair $(a, b)$ is nonhyperbolic in order to estimate the fraction of all pairs between $a_{\mathrm{c}}$ and $a_{\mathrm{h}}$ that are nonhyperbolic. Whatever criterion we propose, we will have errors, both including some that should not be included and excluding some that should be included. Our criterion will be of the following form. We choose a value $\theta_{\text {crit }}$ and count the pair as nonhyperbolic if $\theta_{m}\left(a, b, 10^{4}\right) \leqslant \theta_{\text {crit }}$.

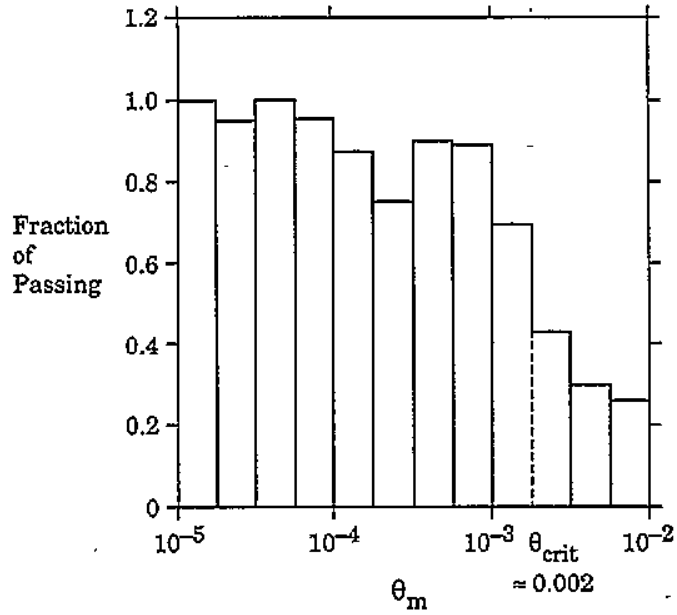

Figure 12. The fraction of parameter values that pass the ten-times-improvement test versus the minimum angle $\theta_{m}\left(a, b, 10^{4}\right)$. 
Is such a test reasonable? As shown in figure 12 , we devide the interval $\left(10^{-5}, 10^{-2}\right)$ into 12 subintervals and for each subinterval we randomly select 50 pairs $(a, b)$ (from our data base) having $\theta_{m}\left(a, b, 10^{4}\right)$ in that subinterval. We now determine ${ }^{2}$ if $\theta_{m}\left(a, b, 10^{7}\right)<$ $\frac{1}{10} \theta_{m}\left(a, b, 10^{4}\right)$. If yes we say that the pair $(a, b)$ passes a ten-times-improvement test. We believe that those passing the ten-times-improvement test are extremely likely to be nonhyperbolic (although we are unable to prove that the chaotic saddle is not hyperbolic with extremely small minimum angle), while almost all those failing are presumably hyperbolic. Figure 12 shows essentially that the smaller $\theta_{m}\left(a, b, 10^{4}\right)$ is, the more likely $(a, b)$ is to pass this test. Based on figure 12, we use the following criterion:

Given a parameter pair $(a, b)$ of the Henon map, if the minimum angle between the stable and unstable manifolds chosen among a trajectory of $10^{4}$ iterates is less than $\theta_{\text {crit }}=0.002$, then the chaotic saddle is counted as nonhyperbolic:

Based on the ten-times-improvement test, the above stated 0.002 criterion for the detection of the nonhyperbolic parameter values has $33 \%$ false negative and $11 \%$ false positive. That is, of these parameter pairs counted as hyperbolic, about $33 \%$ are actually more likely to be nonhyperbolic, while of those pairs counted as nonhyperbolic, about $11 \%$ are more likely to be hyperbolic. Notice that the ten-times-improvement test is too computationally intensive to be used except for the small number of test cases shown in figure 12. We believe that our numerical studies establish for example that the fraction of nonhyperbolic parameter values for ' $a_{\mathrm{c}}<a<a_{\mathrm{h}}$ varies from 0.2 (for $b>0.25$ ) to 1 (for $b$ approaching 0 ). See section 6 for details and section 5 for a theoretical argument predicting this result.

\section{Theoretical model for the fraction of nonhyperbolic parameter values for small $b$}

In this section we develop a theoretical model which predicts that the fraction of parameters in the range between $a_{\mathrm{c}}$ and $a_{\mathrm{h}}$, for which the chaotic saddle is nonhyperbolic, converges to 1 as the Jacobian $b$ goes to 0 . This is a probabilistic model which equates the probability that a particular parmeter is nonhyperbolic to the probability of unbounded growth in a stochastic 'birth-death' process. Integration of this probability over the parameter range of interest then gives $f$, the expected fraction of nonhyperbolic parameter values.

Some facts concerning the geometry of the Hénon map are as follows. There are two fixed points $q$ and $p$ as shown in figure 13(a). For parameters $a$ below $a_{c}$ every point sufficiently close to $U_{a}(p)$, the unstable manifold of $p$, is attracted to $U_{a}(p)$. For parameters above $a_{\mathrm{c}}$ a subset of $U_{a}(p)$ limits on the left branch of $U_{a}(q)$ and under iteration goes to infinity. A parameter value is hyperbolic if all the 'bends' in the curves comprising the closure of $U_{a}(p)$ go to infinity under iteration. If some of these bends do not iterate to infinity then the parameter is nonhyperbolic or there are small bounded attractors. (Since the measure of the set of parameter values for which there exist these small attractors is believed to be negligibly small [15], in this section we ignore the possibility for those small attractors. In fact, as we will demonstrate later, the estimate of the fraction of nonhyperbolic chaotic saddles so obtained in this section agrees reasonably well with our numerical computations.) There are a Cantor set of such bends. These bends are the points

$\dagger$ Note that for nonhyperbolic chaotic saddles, shadowing may fail after $N \sim \varepsilon^{-1 / 2}$ iterates, where $\varepsilon$ is the machine precision [8-10]. In our computation we use $\varepsilon \sim 10^{-14}$, so the maximum number of iterates for which we can make reliable computation is about $10^{7}$. 

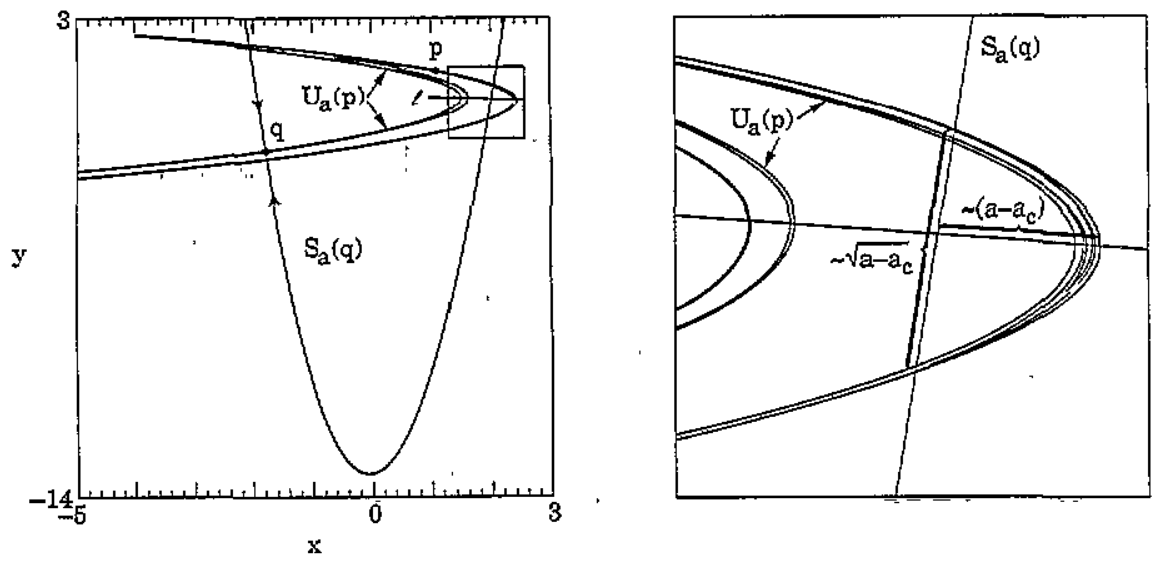

Figure 13. For the Henon map with $a=2$ and $b=.3$. (a)The fixed points $p$ and $q$, the curve $\ell$, the unstable manifold of $p$, and the U-shaped portion of the stable manifold of $q$ are shown for the Hénon map when $a=2$ and $b=0.3$. (b) An enlargement of the small box.

at which maximum bending of the curves of the closure of $U_{a}(p)$ takes place under further iteration and are the analogue of critical points in one-dimensional maps. From here on they will be called the critical points $C$. This definition is rather vague, particularly when the chaotic saddle is nonhyperbolic, for a precise technical definition one should see the work of Benedicks and Carleson [16] (although they apply this concept to the case of chaotic attractors).

The critical points lie on a curve $\ell$ as drawn in figure 13. Thus the set of critical points $C$ is (at least when the chaotic saddle is hyperbolic) the intersection of the curve $\ell$ with the closure of $U_{a}(p)$. To a first approximation this is a Cantor set with $\lambda_{1}{ }^{n}$ pieces of size $\lambda_{2}{ }^{n}$, where $\lambda_{1}$ and $\lambda_{2}$ are the two Lyapunov numbers for the chaotic saddle. We fix our parameter $b$ to be a small positive number and our parameter $a$ to be between $a_{\mathrm{c}}$ and $a_{\mathrm{h}}$. In this parameter range the two Lyapunov numbers for the chaotic saddle are approximately $\lambda_{1} \approx 2$ and $\lambda_{2} \approx \frac{b}{2}$.

The behavior of the critical points under iteration can be seen as follows. Those critical points which on some iteration lie outside the U-shaped region bounded by the part of the stable manifold $S_{a}(q)$ depicted in figure $13(a)$ will limit on the left branch of $U_{a}(q)$ under iteration and thus go to infinity. Critical points which are sufficiently close to each other so that on a given iteration they can all fall to the right of $S_{a}(q)$ in the small box depicted in figure $13(a)$ are treated as effectively one critical point for that iteration. As the critical points are iterated, on the average they locally separate at an exponential rate close to $\ln \left(\lambda_{1}\right)$. These critical points are originally more or less a Cantor set with $\lambda_{1}{ }^{n} \approx 2^{n}$ pieces of size $\lambda_{2}{ }^{n} \approx\left(\frac{b}{2}\right)^{n}$. Consider a piece of size $(b / 2)^{n}$, after $n(\ln 2-\ln b) /(\ln 2)$ iterations its size has lengthened to be of order 1 . So we see that the number of effective critical points grows at an exponential rate of $(\ln 2)^{2} /[\ln 2-\ln b]$. If a representative critical point contained in an effective critical point lies to the right of $S_{a}(q)$ in the small box on a given iteration, we assume that all the critical points in that effective critical point likewise lie to the right of $S_{\mathfrak{a}}(q)$ and on further iteration go to infinity.

We now describe the 'birth-death' process which we use to heuristically estimate the probability that the parameter $a$ is nonhyperbolic. Since the number of effective critical points grows at an exponential rate of approximately $(\ln 2)^{2} /(\ln 2-\ln b)$ and for small $b$ values this rate is small, we assume that on any given iteration each effective critical point 
has a probability of order $(\ln 2)^{2} /[\ln 2-\ln b]$ of stretching into two effective critical points (birth). In addition, we assume, on each iteration, that each effective critical point which has not yet fallen outside of the U-shaped region will fall along a curve of $U_{a}(p)$ in a random place inside the union of the U-shaped region and the small box. Therefore, since the parabolic curves comprising the part of $U_{a}(p)$ in the small box have lengths of order $\sqrt{a-a_{c}}$, we have that on any iteration each effective critical point has probability of order $\sqrt{a-a_{\mathrm{c}}}$ of falling outside of the U-shaped region (death). So, for each effective critical point there is a probability of 1 minus a term of order $\sqrt{a-a_{c}}+(\ln 2)^{2} /[\ln 2-\ln b]$ that the effective critical point neither stretches into two effective critical points nor falls outside of the U-shaped region. We assume that each effective critical point behaves independently of all other effective critical points on each iteration, and thus this is a classic 'birth-death' process.

We now calculate $G$, the probability that the effective critical points all eventually iterate to infinity given that we start with exactly one effective critical point (that is, we calculate the probability that the parameter is hyperbolic). Since we assume that each effective critical point behaves independently of all others on each iteration, we see that the probability that the effective critical points all eventually iterate to infinity given that we start with exactly two effective critical points is $G^{2}$. Also, the probability that a given effective critical point stretches into two before it falls outside the U-shaped region is of order

$$
H=\frac{(\ln 2)^{2}}{(\ln 2)^{2}+(\ln 2-\ln b) \sqrt{a-a_{c}}} .
$$

Using conditional probabilities, we can write $G=(1-H)+H G^{2}$, which we solve to find the probability that

$$
G=\frac{(1-H)}{H}=\frac{\ln 2-\ln b}{(\ln 2)^{2}} \sqrt{a-a_{\mathrm{c}}} .
$$

We now estimate the fraction of hyperbolic parameter values between $a_{\mathrm{c}}$ and $a_{\mathrm{h}}$ by averaging $\left(\frac{\ln 2-\ln b}{(\ln 2)^{2}} \sqrt{a-a_{\mathrm{c}}}\right)$ for parameters $a$ between $a_{\mathrm{c}}$ and $a_{\mathrm{h}}$, bearing in mind that $a_{\mathrm{h}}-a_{\mathrm{c}}$ is of order $b$. That is, we compute

$$
\frac{1}{b} \int_{0}^{b} \frac{\ln 2-\ln b}{(\ln 2)^{2}} \sqrt{x} \mathrm{~d} x=\frac{2(\ln 2-\ln b)}{3(\ln 2)^{2}} \sqrt{b}
$$

and thus the fraction of hyperbolic parameter values is of order $-\sqrt{b} \ln \left(\frac{b}{2}\right)$. Since the limit as $b$ decreases to 0 of $-\sqrt{b} \ln \left(\frac{b}{2}\right)$ is 0 , we see that the predicted fraction of hyperbolic parameter values goes to 0 . Therefore, the predicted fraction of nonhyprerbolic parameter values goes to 1 as $b$ goes to 0 . Figure $15(b)$ illustrates the numerical evidence which supports the contention that the nonhyperbolic fraction goes to 1 as $b$ goes to 0 .

\section{Conclusions: fraction of nonhyperbolic parameter values and the size of Newhouse intervals}

Based on the criterion in section 4, we have calculated the nonhyperbolic parameter values $(a, b)$ for $b \in(0,1)(|J|=b)$ and $a \in\left[a_{\mathrm{c}}(b), a_{\mathrm{h}}(b)\right]$ of the Henon map. The result is shown in figure 5 , where the dots denote the nonhyperbolic parameter values. The fraction 


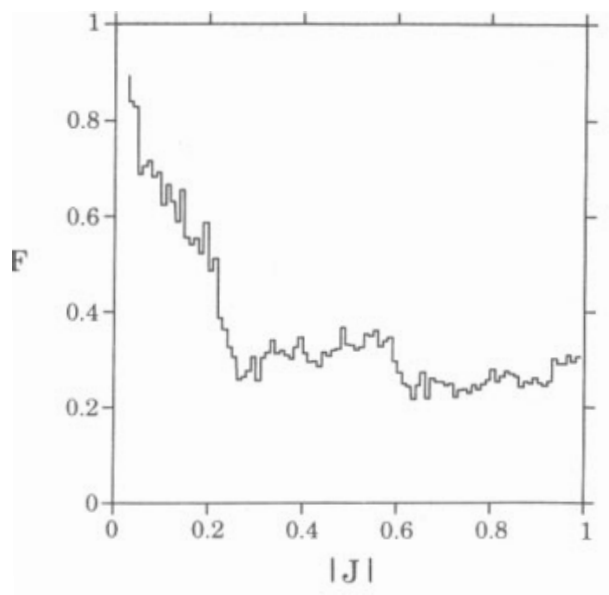

Figure 14. Fraction of nonhyperbolic paraneter values versus $|J|(=b)$ for $a_{\mathrm{c}}(b)<a<a_{\mathrm{h}}(b)$. Note that for all the $|J|$ values considered, the fraction of nonhyperbolic parameter values is larger than 0.2 . We have not plotted the fraction when $|J|<0.03$. See next figure for the range $0<|J|<0.03$.
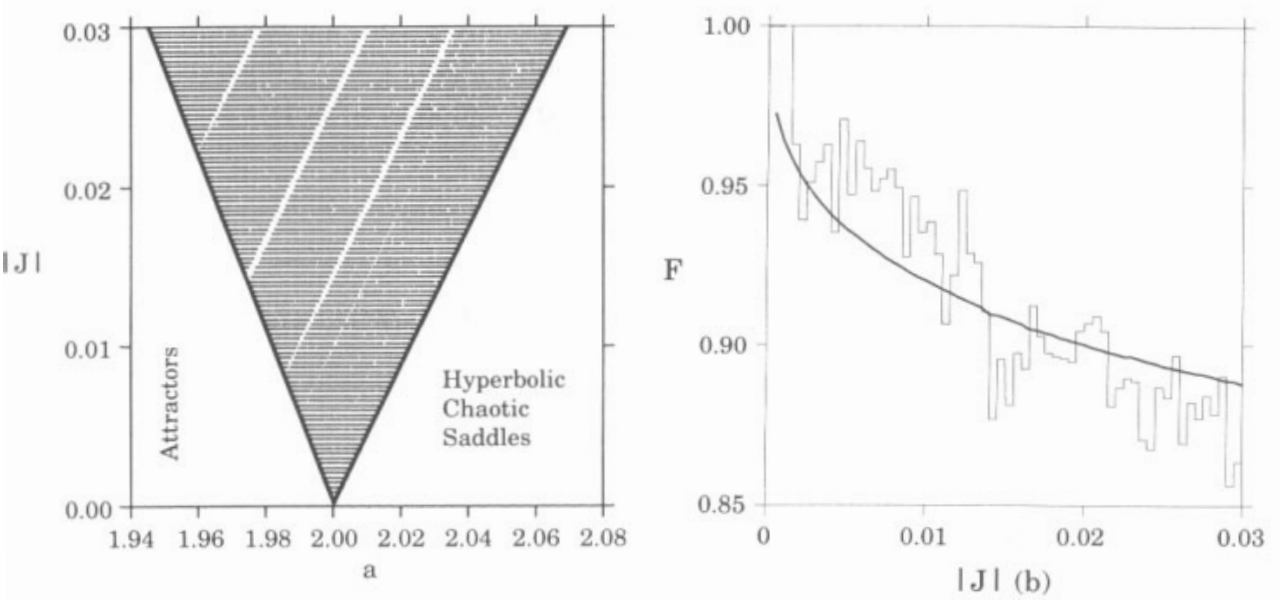

Figure 15. (a) Nonhyperbolic parameter values for $0<|J|<0.03$ and $a_{\mathrm{c}}<a<a_{\mathrm{h}}$. (b) The fraction of nonhyperbolic parameter values for small Jacobian $(0<|J|<0.03)$. Note that the fractions are close to one. The continuous curve in the figure derives from a heuristic argument of section 5 for small Jacobian.

of nonhyperbolic parameter values for $b \in(0,1)$ is shown in figure 14 where we see that for each value of $b$ considered, at least $20 \%$ of the parameter pairs tested are nonhyperbolic for $a_{\mathrm{c}}(b)<a<a_{\mathrm{h}}(b)$.

The most striking feature of figure 14 is that for small values of the Jacobian, the fraction of nonhyperbolic parameter values is close to one. Figure $15(a)$ shows the nonhyperbolic parameter values for $0<|J|<0.03$ and $a_{c}<a<a_{\mathrm{h}}$. Figure $15(b)$ shows the fraction of nonhyperbolic parameter values for $0<|J|<0.03$. Based on our numerical studies and the heuristic argument in section 5 , we conjucture that the fraction of nonhyperbolic parameter values tends to 1 as $|J| \rightarrow 0$.

Figures $16(a)$ and $16(b)$ show the distribution of our calculated Newhouse intervals with length larger than 0.02 and 0.04 , respectively. For each $b$, an interval is plotted if all $a$ in that interval tested positive and the length of the inteval is at least 0.02 [figure $16(a)$ ] or 0.04 [figure $16(b)$ ]. We have also calculated the maximum Newhouse intervals for all the $b$ values considered. We find that the maximum Newhouse interval is large (about 0.12 ). This 

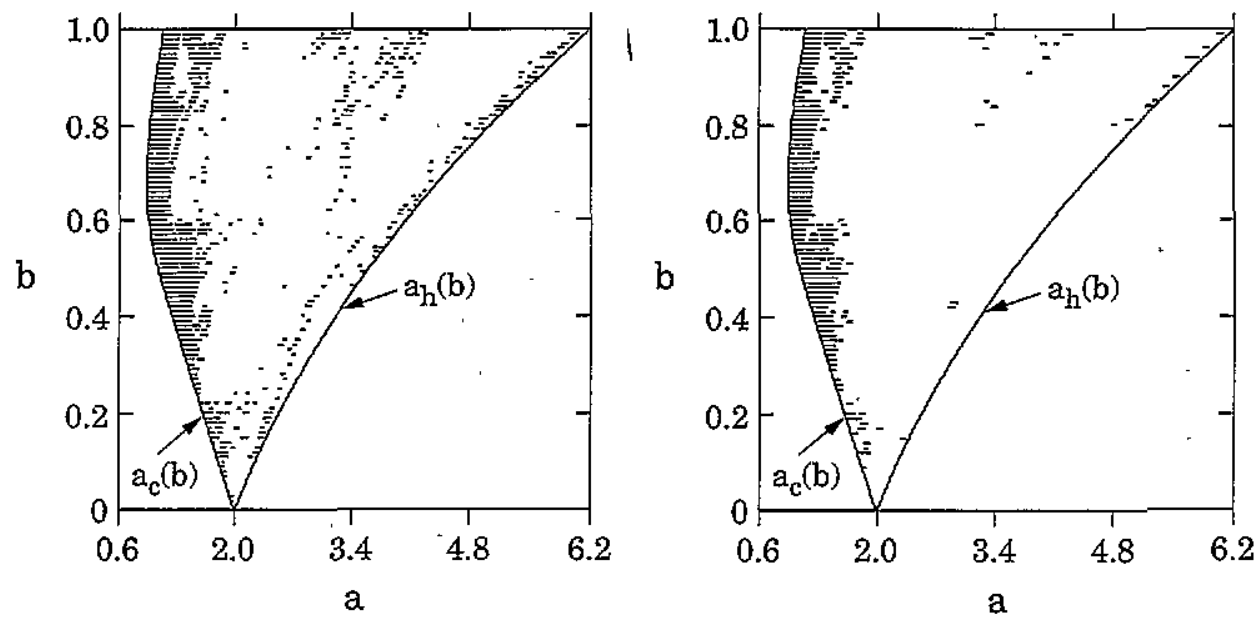

Figure 16. Computed Newhouse intervals with (a) length $>0.02$, and $(b)$ length $>0.04$.

is surprising because it was believed previously that the Newhouse intervals are typically small [17] (of order $10^{-6}$ ).

While it is important to estimate the size of Newhouse intervals, we cannot guarantee that what appears to be a Newhouse interval is one in fact. We studied two values of $b$ in detail, $b=0.3$ and $b=0.7$. Our procedure is as follows. Given a value $b$, we choose a gxid of 700 values of $a$ in $\left(a_{\mathrm{c}}, a_{\mathrm{h}}\right)$. For each $a$ we compute $\theta_{m}\left(a, N=10^{4}\right)$. We choose the largest interval for which all the tested $a$ values are nonhyperbolic according to our criterion discussed in section 4 . We call this interval an 'apparent Newhouse interval'. Obviously there could be small undetected gaps of hyperbolic parameter values in such an interval. To increase the plausibility of our suggestion that we actually have a Newhouse interval, we decrease the grid size by a factor of 10 in the apparent Newhouse interval. We then use the criterion discussed in section 4 to test whether the new parameter values are also nonhyperbolic. If one of these values should fail the test, we perform the ten-timesimprovement test for this parameter value. In other words, we compute further upto $10^{7}$ iterates of the map to see whether the minimum angle can be ten times smaller than that obtained using $10^{4}$ iterates. Some parameter values that fail our criterion would still fail the ten-times-improvement test. Hence, they are apparently hyperbolic parameter values. However, we found those values to be always near the ends of the apparent Newhouse interval. Thus, our testing procedure only results in small decreases in the length of the apparent Newhouse interval.

Reader beware. We predict that more rigorous investigation will validate our numerical findings that nonhyperbolic chaotic saddles are common, especially for small Jacobian. Nonetheless, chaotic saddles can have complicated structure on many different scales and this complexity is hinted at by the histograms of angles shown in figures $1(b)$ and $2(b)$. Therefore, there is currently no rigorous assurance of the reliability of our estimate of the fraction of nonhyperbolic parameter values.

\section{Acknowledgments}

We thank Dr H E Nusse for valuable discussions and Dr J Guckenheimer for numerous 
comments. This work was supported by the US Department of Energy (Scientific Computing Staff, Office of Energy Research) and the Air Force (Air Force Office of Scientific Research). One of us (I Kan) is supported by AFOSR.

\section{Appendix. PIM-triple method}

In this appendix, we briefly describe the PIM-triple method $[12,13]$ to find a trajectory on chaotic saddles existing in a given region for a diffeomorphism $F: R^{2} \rightarrow R^{2}$. A PIM(Proper Interior Maximum) triple is three points $(a, c, b)$ in a straight line segment $L$ such that the interior point $c$ (i.e., $c$ is between $a$ and $b$ ) has an escape time (time for leaving the region) that is larger than the escape times of both $a$ and $b$, that is, $(a, c, b)$ has a proper interior maximum.

The steps of the PIM-triple procedure are as follows:

1. Specify a region of interest. If there is (are) attractor(s) coexisting in the region, isolate the attractor(s) with circles of appropriate radii. If a trajectory asymptotes to any attractor, this trajectory is considered to have escaped from the region.

2. Choose a line segment $L_{0}$ in the above region that straddles the stable manifold of the chaotic saddle. Distribute uniformly a number (say, 30) of points on the line segment and calculate the escape time for each point. Choose any PIM triple $(a, c, b)$ from the 30 points (Do not include both end points of the original interval). In principle, the number 30 may have to be increased but that was not necessary in this study. There are often many PIM-triples that can be selected. One PIM-triple can be chosen at random, or one can choose one systematically, choosing $c$ to have the largest escape time.

3. It is known [12] that PLM-triple trajectories in the plane can be generated for arbitrarily many iterates, that is for $n$ (the length of the trajectory) as large as desired. For nonhyperbolic systems, we occasionally encounter a segment $L_{n}$ that has no PLM-triple. In this case, we back up to, say, $L_{n-5}$ and choose a different PIM-triple.

4. Use the PIM-triple as the new line segment and repeat step (2) until the length of the PIM-triple so obtained is less than, say, $10^{-9}$ (denoted by $I_{0}$ ). Write $I_{0}=R\left(L_{0}\right)$, that is, $I_{0}$ is our refined $L_{0}$.

5. Iterate the end points of $I_{0}$ forward under the map. The two images are end points of a new segment denoted by $L_{1}$. If the length of $L_{1}$ exceeds $10^{-9}$, we apply step (3) until its length is less than $10^{-9}$ and we then replace $L_{1}$ by $R\left(L_{1}\right)$.

6. Repeat step (4) to find a sequence of PIM-triple intervals $\left[L_{n}\right]_{n \geqslant 0}$.

7. Write $x_{n}$ for the midpoint of $L_{n}$. It follows that $x_{n+1}=F\left(x_{n}\right)+\varepsilon_{n}$, where $\varepsilon_{n}$ is typically on the order of $10^{-9}$.

\section{References}

[1] Grebogi C, Ott E and Yorke J A 1983 Crises, sudden changes in chaotic attractors, and transient chaos Physica 7D 191-200

[2] Grebogi C, Ott E, Romeiras F and Yorke J A 1987 Critical exponents for crisis-induced intermittency Phys. Rev. A 36 5365-80

[3] Newhouse S E 1979 The abundance of wild hyperbolic sets and non-smooth stable sets for diffeomorphisms Publ. Math. IHES 50 101-51

[4] Robinson C 1983 Bifurcation to infinitely many sinks Commun. Math. Phys. 90 433-59

[5] Anosov D V 1967 Geodesic flows and closed Riemannian manifolds with negative curvature Proc. Steklov Inst. Math. 901

[6] Bowen R $1975 \omega$-limit sets for Axiom A diffeomorphisms J. Diff. Eq. 18333 
[7] Grebogi C, Ott E and Yorke I A 1988 Unstable periodic orbits and the dimension of multifractal chaotic attractors Phys. Rev. A 37 1711-24.

[8] Hammel S M, Yorke J A and Grebogi C 1987 Do numerical orbits of chaotic dynamical processes represent true orbits? J. Complexity 3 136-45

[9] Hammel S M, Yorke J A and Grebogi C 1988 Numerical orbits of chaotic processes represent true orbits Bull. Am Math. Soc. $19465-9$

[10] Grebogi C, Hammel S M, Yorke J A and Sauer T 1990 Shadowing of physical trajectories in chaotic dynamics: containment and refinement Phys. Rev. Lett. 65 1527-30

[11] Hammel S M 1990 Phys. Lett. 148A 421

[12] Nusse H E and Yorke J A 1989 A procedure for finding numerical trajectories on chaotic saddles Physica 36D 137-56

[13] Hsu G, Ott E and Grebogi C 1988 Strange saddles and the dimension of their invariant manifolds Phys. Lett. 127A 199-204

[14] You Z P, Kostelich E and Yorke J A 1991 Calculating stable and unstable manifolds Int. J. Bifurcation Chaos 1 605-23

[15] Tedeschini-Lalli L and Yorke J A 1985 How often do simple dynamical processes have infinitely many coexisting sinks? Commun. Math Phys 106 635-57

[16] Benedicks $M$ and Carleson $L 1991$ The dynamics of the Henon map Ann, Math. 133 73-169

[17] Kan I, Koçak H and Yorke J A 1991 Persistent homoclinic tangencies in the Hénon family Preprint 